\title{
Fluorinated microemulsions as reaction media for fluorous nanoparticles $\dagger$
}

\author{
Received 26th October 2009, Accepted 24th November 2009 \\ First published as an Advance Article on the web 4th January 2010 \\ DOI: $10.1039 / \mathrm{b} 922260 j$
}

Martin J. Hollamby, ${ }^{a}$ Julian Eastoe, ${ }^{* a}$ Kevin J. Mutch, ${ }^{a}$ Sarah Rogers ${ }^{b}$ and Richard K. Heenan ${ }^{b}$

\begin{abstract}
New fluorinated microemulsions (F-MEs) formulated from partially fluorinated solvent-co-solvent mixtures and fluorinated anionic AOT-analogue surfactants are reported. These F-MEs permit incorporation of water into fluorinated solvents, up to a volume fraction $\phi_{\text {water }} \approx 0.13$. Interestingly, phase behavior and structures, determined by small-angle neutron scattering (SANS), parallel many other classical AOT-type microemulsions, both in normal hydrocarbon and in supercritical- $\mathrm{CO}_{2}$ $\left(\mathrm{scCO}_{2}\right)$ solvents. Using these new F-MEs as reaction media, F-capped silver nanoparticles (Ag-NPs) have been synthesized, representing the first reported preparation of F-NPs in fluorous phase microemulsions.
\end{abstract}

\section{Introduction}

Water-in-fluorocarbon (w/fc) microemulsions (also called F-MEs) combine the high oxygen carrying capacity of fluorocarbon solvents ${ }^{1}$ with an ability to stabilize hydrophilic drug molecules, and as such are potentially useful drug delivery systems. ${ }^{2-5}$ These reversed-curvature F-MEs are typically stabilized by F-surfactants, such as perfluorinated alkylphosphates, ${ }^{\mathbf{4}}{ }^{6}$ oxosulfonylisocyanates, ${ }^{7}$ non-ionics,${ }^{8-10}$ carboxylic acids, ${ }^{10,11}$ and polymers. ${ }^{12-14}$ High-pressure F-MEs are also known, with the fluoro-hydrocarbon refrigerant R134a as continuous solvent, stabilized by a fluorinated derivative of the common surfactant AOT (sodium bis(2-ethylhexyl)sulfosuccinate). ${ }^{15}$ Fluorocarbons have additionally found extensive application in biphasic catalysis, ${ }^{16}$ permitting both high activity and selectivity. ${ }^{16,17}$ Such benefits can also be found when using only a single fluorocarbon phase. ${ }^{5,18}$ The ability to disperse inorganic and noble metal nanoparticles (NPs), which are known efficient catalysts, ${ }^{19,20}$ in fluorinated media would be of particular interest, as high $\mathrm{O}_{2}$ loadings could be achieved in the continuous F-solvent for oxidation reactions. One strategy to achieve fluorous NPs would be through classical microemulsion-based synthesis, ${ }^{21,22}$ which has already been extensively applied for NP preparation in ambient and high-pressure alkanes and also liquid/supercritical$\mathrm{CO}_{2}\left(\mathrm{scCO}_{2}\right) \cdot{ }^{19,21,23}$ However, to date microemulsion NP synthesis has yet to be reported in F-MEs. Whilst aqueous methods can be employed to form F-capped NPs, ${ }^{24}$ using F-MEs as the reaction medium technique has advantages for accessing a wider range of materials, and offers greater control over particle characteristics. ${ }^{21,22}$ Hence there are good reasons for expanding the palette of ME systems suitable for NP synthesis to

\footnotetext{
${ }^{a}$ School of Chemistry, University of Bristol, Cantock's Close, Bristol, UK BS8 1TS. E-mail: Julian.Eastoe@bristol.ac.uk

${ }^{b}$ ISIS-CCLRC, Rutherford Appleton Laboratory, Chilton, Oxon, UK $O X 110 Q X$

$\uparrow$ Electronic supplementary information (ESI) available: Details of calculations and model fitting; SANS profiles and analysis for F-microemulsions not shown in the main paper; justification for high polydispersity used in data fitting; SAED micrographs and analysis; UV profile of redispersed nanoparticles. See DOI: 10.1039/b922260j
}

include fluorinated microemulsions; this paper describes such developments.

Here, fluorinated AOT-analogue F-surfactants have been employed as stabilizers. The popular H-AOT is based on a dichain sulfosuccinate motif, which is a very flexible and adaptable molecular template. As such common H-AOT finds extensive use in microemulsion-based NP synthesis. ${ }^{21}$ The related F-AOT surfactants have been employed to stabilize other systems, in which H-AOT is inactive, such as water-in- $\mathrm{scCO}_{2}\left(\mathrm{w} / \mathrm{scCO}_{2}\right)$ microemulsions ${ }^{25}$ and $\mathrm{CuS}$ nanoparticles in pure $\mathrm{scCO}_{2} \cdot{ }^{26}$ Hence such F-surfactants appear to be ideal candidates for stabilizing F-MEs and fluorous NP dispersions.

This work introduces a new set of four-component F-MEs, and demonstrates applications for generating F-capped nanoparticles under bench-top conditions. The main solvent employed is $2 \mathrm{H}, 3 \mathrm{H}$-perfluoropentane (HPFP, a high boiling point industry model for $\mathrm{R} 134 \mathrm{a}),{ }^{27,28}$ with $1 H, 1 H, 5 H$-octafluoropentanol (OFP) added as a co-solvent. The F-AOT surfactants used are sodium bis( $1 H, 1 H, 5 H$-octafluoropentyl)-2sulfosuccinate (diHCF4) and sodium bis( $1 H, 1 H$-perfluorobutyl)-2-sulfosuccinate (diCF3), which were initially designed, synthesized and employed to stabilize w/ $\mathrm{scCO}_{2}$ microemulsions. ${ }^{29}$ In addition here, a diCF3-stabilized water-in$1 H$-perfluorohexane $(\mathrm{HPFH})$ system was investigated to shed light on solvent effects with F-MEs. It was also found possible to form microemulsions using the surfactant diCF4, but studies were limited by the availability of material. Temperaturedependent microemulsion phase behavior has been documented for these systems, and domain structure has been investigated by small-angle neutron scattering (SANS).

To exhibit potential applications of these new F-MEs, silver nanoparticles (Ag-NPs) have been synthesized in diCF3-stabilized systems. These F-capped Ag-NPs were characterized by UV-vis, transmission electron microscopy (TEM) and highresolution TEM (HR-TEM). The prepared Ag-NPs can also be recovered from the F-MEs and redispersed in another fluorinated solvent. Such F-surfactant stabilized NPs could have applications in fluorocarbon-based catalysis or novel delivery systems. In addition, since F-compounds are known to have high $\mathrm{CO}_{2}$ compatibility, stabilizing $\mathrm{scCO}_{2}$ microemulsions, ${ }^{30,31}$ and 
dispersing inorganic NPs in $\mathrm{CO}_{2},{ }^{32}$ this F-ME-based method could provide new routes to generating $\mathrm{CO}_{2}$-compatible NPs, improving on existing approaches. ${ }^{24}$ Given the high solubility of $\mathrm{O}_{2}$ in F-solvents, and also the known solubility of F-surfactants and $\mathrm{F}$-ligands in $\mathrm{scCO}_{2}$, this could open up new opportunities for catalysis in such challenging low dielectric solvent environments.

\section{Results and discussion}

\section{Phase behavior}

The three w/fc microemulsion systems studied were diHCF4/ $2 H, 3 H$-perfluoropentane (HPFP), diCF3/HPFP, and diCF3/1 $H$ perfluorohexane (HPFH). Three-component systems did not form microemulsions, and in order to achieve clear single phases, a fluorinated alcohol co-surfactant was required $(1 \mathrm{H}, 1 \mathrm{H}, 5 \mathrm{H}$ octafluoropentanol, OFP). The co-surfactant to solvent volume ratio was kept constant at $1: 9$ (10 vol $\%$ co-solvent). Phase diagrams at constant surfactant concentration $(100 \mathrm{mM})$ were determined as a function of temperature and water content, $w=$ [water]/[surfactant], in order to locate phase boundaries. The phase diagram for the diHCF4/HPFP system is shown in Fig. 1B, diCF3/HPFH in Fig. 1C and diCF3/HPFP in Fig. 1D. The visual appearance of the different phase regions is exemplified in Fig. 1A (cases i-iv). It should be noted that the surfactants employed are efficient, permitting microemulsification of water up to $w=70$, which represents a water volume fraction $\phi_{\text {water }} \approx$ 0.13 at [surfactant] $=100 \mathrm{mM}$. Unfortunately, at higher temperature phase stability is limited owing to the low boiling points of these volatile F-solvents (HPFP bp $53.5^{\circ} \mathrm{C}, \mathrm{HPFH}$ bp $71^{\circ} \mathrm{C}$ ).

All phase diagrams are funnel shaped and temperature sensitive, similar to the commonly studied H-AOT-stabilized waterin-hydrocarbon oil systems. ${ }^{33,34}$ Three distinct regions are observed: at lower temperatures and $w$ values between 15 and 70 two-phase Winsor II systems appear, consisting of transparent thin upper aqueous phases and lower fluorocarbon-rich phases (case i, Fig. 1A). Increasing temperature leads to the formation of single-phase $\mathrm{L}_{2}$ microemulsions (case ii, Fig. 1A). This stable region spans a much greater temperature range for diCF3stabilized systems than for diHCF4/HPFP, suggesting that a shorter, fully fluorinated-tail surfactant is more efficient for microemulsion formation.

Interestingly, solvent type seems to have only a slight influence on the stability of diCF3-stabilized systems; phase boundaries are shifted to lower temperature by a few degrees for the shorter chain HPFP (five carbons) compared to that for HPFH (six carbons). This is in marked contrast to comparable phase behavior of AOT-stabilized systems in hydrocarbon oils where strong effects of alkane chain length are observed. ${ }^{27,28}$ However, the comparisons should not be pushed too hard, since there are other structural differences between HPFP and HPFH than merely chain length alone.

Further increase in temperature results in microemulsion phase failure. Here, the surfactants differ in behavior: the
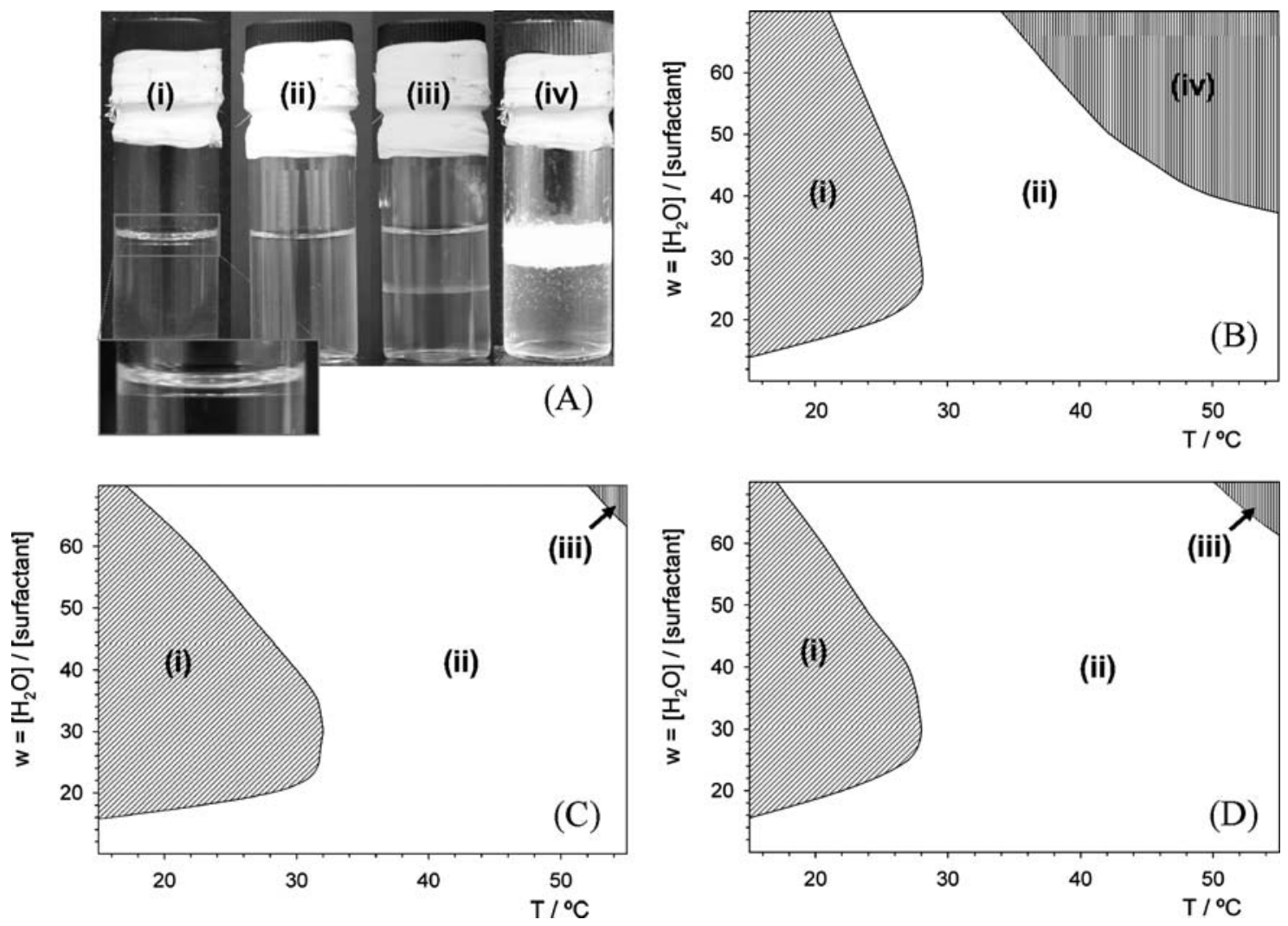

Fig. 1 (A) Visual appearance of samples i, ii, iii, and iv; temperature-dependent phase behavior (B) for diHCF4/HPFP; (C) for diCF3/HPFH and (D) for diCF3/HPFP systems. In all cases, [surfactant] $=100 \mathrm{mM}$, with addition of co-surfactant (OFP, 10 vol\%). Single transparent phases (case ii) are denoted by the white regions, shaded regions (cases i, iii, iv) represent unstable systems. 
diHCF4/HPFP system (case iv, Fig. 1A) splits into two distinct phases, an opaque, white upper phase with a translucent lower phase; whereas both diCF3-stabilized systems (case iii, Fig. 1A) show a liquid-liquid critical-type separation likely to consist of two coexisting microemulsions. ${ }^{35}$ Unfortunately, solvent evaporation precluded further analyses of these systems.

\section{Small-angle neutron scattering}

SANS measurements were carried out in single-phase regions of the different systems. Example SANS profiles for diCF3/HPFH microemulsion droplets are shown as a function of $w$ in Fig. 2, and as a function of [diCF3] in Fig. 3. Other SANS profiles are given in the ESI $\dagger$ (Fig. S2-S7). In a $\log I(Q) v s . \log Q$ representation, these profiles in general show $Q^{0}$ dependence at low $Q$ and $Q^{-4}$ dependence at high $Q$, indicating that droplet cores are

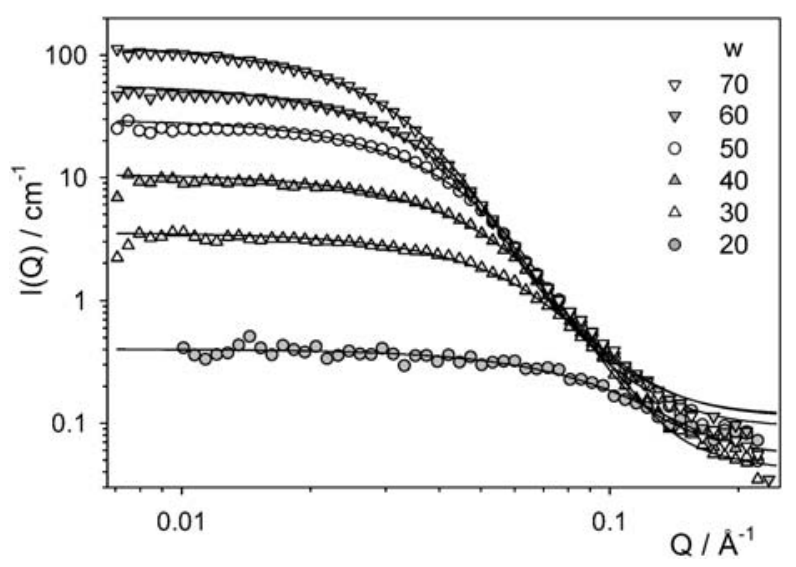

Fig. 2 Core-contrast SANS data from $\mathrm{D}_{2} \mathrm{O} / \mathrm{diCF} 3 / \mathrm{OFP} / \mathrm{HPFH}$ microemulsions at different $w=\left[\mathrm{D}_{2} \mathrm{O}\right] /[\mathrm{diCF} 3]$. Solid lines represent form factor fits to the Schultz polydisperse spheres model. [diCF3] $=100 \mathrm{mM}$, OFP : HPFH $=1: 9(\mathrm{v} / \mathrm{v}), T=40^{\circ} \mathrm{C}$.

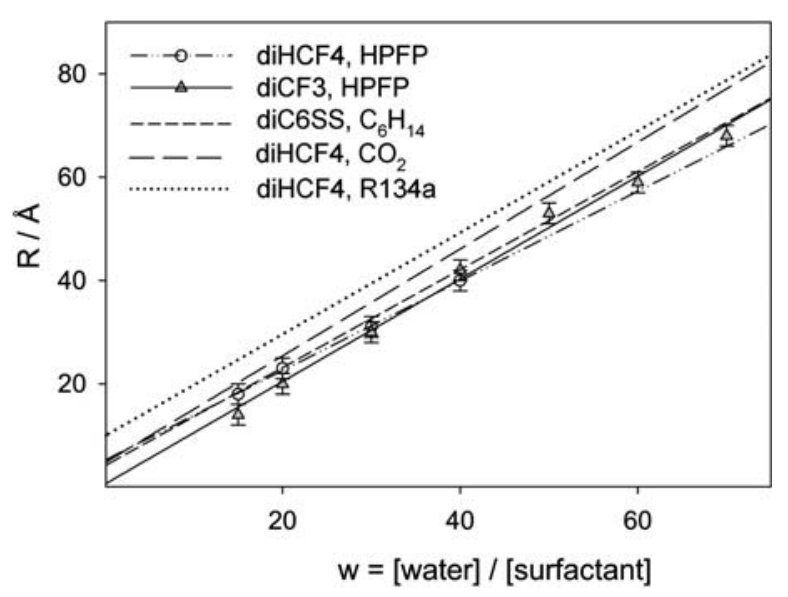

Fig. 3 Water droplet radius $R_{\mathrm{c}} v s . w$ for diHCF4- and diCF3-stabilized water/OFP/HPFP microemulsions. $R_{\mathrm{c}}$ values are calculated from SANS analysis of core contrasts (Tables 1 and 2), corrected for polydispersity (eqn (1) and (2)). Example error bars are shown. Linear fits from these data, in addition to those for data from diC6SS-stabilized water/hexanol/ hexane ${ }^{33,34}$ and diHCF4-stabilized water/ $\mathrm{CO}_{2}{ }^{29}$ and water/R134a microemulsion systems ${ }^{15}$ are shown. essentially spherical with a sharp interface. Data were fitted to a model representing non-interacting Schultz polydisperse spheres; the fitted parameters are summarized for diCF3 in Table 1 and diHCF4 in Table 2. Fits are characterized by the requirement for a high polydispersity (see ESI $\dagger$ ). One interesting point is that the absolute intensities did not fit with the expected scale factors based on scattering length density differences (Table $\mathrm{S} 1 \dagger$ ) and droplet volume fractions calculated from known sample compositions. This has been seen before in hydrocarbon systems, ${ }^{36}$ there $\mathrm{H}-\mathrm{D}$ exchange from alcohol co-surfactant $(\mathrm{H})$ to $\mathrm{D}_{2} \mathrm{O}$ in droplet cores was suggested to account for reduced SANS intensities. This effect alone cannot fully account for the discrepancies, as scale factors are consistently $70-90 \%$ of theoretical value regardless of droplet contrast $\left(\mathrm{H}_{2} \mathrm{O}\right.$ or $\left.\mathrm{D}_{2} \mathrm{O}\right)$ used. This could be an artifact of high polydispersities as there is close coupling in the Schultz fitting model between scale factor, droplet radius, $R_{\mathrm{c}}$, and polydispersity, $p$. Furthermore, the unknown contrast of the surfactant layer and/or a finite solubility of water in the fluorinated continuous layer (or inclusion of F-alcohol OFP into the water droplet) may serve to compound these effects. Bearing these issues in mind, there are obviously limitations to consider, and the reported absolute values for $R_{\mathrm{c}}$ and $p$ should be taken as approximate $( \pm 10 \%)$. However, the

Table 1 Average radii, $R_{\mathrm{c}}$, and polydispersity, $p$, from SANS profile fitting for diCF3-stabilized F-MEs. Typical uncertainties for both parameters are of order $10 \%$. Numbers in brackets represent freshly made repeat samples

\begin{tabular}{llccc}
\hline Solvent & {$[\mathrm{diCF}] / \mathrm{mM}$} & $w$ & \multicolumn{1}{c}{$R_{\mathrm{c}} / \AA$} & $p$ \\
\hline HPFH & 100 & 15 & 12 & 0.28 \\
& & 20 & $15(16)$ & 0.28 \\
& & 30 & $25(24)$ & 0.28 \\
& & $32(31)$ & 0.28 \\
& & 50 & 39 & 0.28 \\
& 75 & 70 & 46 & 0.28 \\
HPFP & 50 & 40 & 50 & 0.28 \\
& 100 & 40 & 22.5 & 0.34 \\
& & 15 & 12 & 0.49 \\
& & 20 & $17(17)$ & 0.28 \\
& & 30 & 26 & 0.28 \\
& & 40 & $36(36)$ & 0.28 \\
& 50 & 60 & 46 & 0.28 \\
& & 70 & 51 & 0.28 \\
& & 40 & 34 & 0.28 \\
& & 60 & 50 & 0.33 \\
& & &
\end{tabular}

Table 2 Average radii, $R_{\mathrm{c}}$, and polydispersity, $p$, from SANS profile fitting for diHCF4-stabilized F-MEs. Also given are $S(Q)$ parameters $\kappa$, being related to the strength of interactions via the isothermal compressibility and $\zeta$ a correlation length. Typical uncertainties for both parameters are of order $10 \%$

\begin{tabular}{lllll}
\hline$w$ Value & $R_{\mathrm{c}} / \AA$ & $p$ & $\kappa$ & $\zeta / \AA$ \\
\hline 15 & 16 & 0.28 & - & - \\
20 & 20 & 0.28 & - & - \\
30 & 27 & 0.28 & 1.0 & 38 \\
40 & 34 & 0.28 & 2.3 & 47 \\
\hline
\end{tabular}


general trends in system behavior as a function of composition and chemical identity are clearly highlighted.

Influence of $w$-ratio. At constant surfactant concentration, an increase in $w$ is observed to lead to an increase in droplet core radius. This droplet swelling is in agreement with findings from other fluorinated systems $\mathrm{s}^{12-14}$ and sulfosuccinate-stabilized hydrocarbon-based systems. ${ }^{33,34}$ The latter are known to obey a swelling law ${ }^{15,29,33,34}$ as given in eqn (1).

$$
\begin{aligned}
& \alpha(p) R_{\mathrm{c}}^{\mathrm{av}}=\frac{3 \nu_{\mathrm{w}}}{a_{\mathrm{h}}}+\frac{3 \nu_{\mathrm{h}}}{a_{\mathrm{h}}} \\
& \alpha(p)=1+2 p^{2}
\end{aligned}
$$

In the above $p$ is a fitted polydispersity, $\nu_{\mathrm{w}}$ is the molecular volume of water and $v_{\mathrm{h}}$ is the head group volume. Therefore, a plot of droplet radius $R_{\mathrm{c}}$ against $w$ value should fit a straight line, with gradient inversely proportional to the effective headgroup area in the interfacial film $a_{\mathrm{h}}$. The swelling law is given for diCF3/HPFP and diHCF4/HPFP systems (Fig. 3), alongside the relationships for comparable systems obtained from literature; diHCF4-stabilized w/scCO 2 microemulsion droplets, ${ }^{29}$ diHCF4stabilized water-in-high-pressure fluorocarbon R134a microemulsion droplets, ${ }^{15}$ and a directly analogous quaternary hydrocarbon-only system, water/diC6SS/hexanol/hexane..$^{33,34}$ Calculated $a_{\mathrm{h}}$ values are $91 \AA^{2}$ and $103 \AA^{2}$ for diCF3- and diHCF4-stabilized OFP/HPFP systems respectively, comparing well to those for diHCF4-stabilized w/scCO $\left(115 \AA^{2}\right)$ and w/R134a $\left(90 \AA^{2}\right.$ ) systems (characteristic uncertainties $\pm 10 \AA^{2}$ ). This underlines the wide applicability of sulfosuccinate surfactants: by changing only the tail structure, a family of amphiphiles has been assembled which are compatible with a wide range of very different non-polar solvents. Furthermore, there are no significant effects on the underlying interfacial or aggregation structures in these very different microemulsions.

Influence of other composition parameters. There is little difference between $p$ and $R_{\mathrm{c}}$ values found in diCF3- or diHCF4stabilized microemulsions (Fig. 3). The only minor change in SANS profiles is an additional attractive $S(Q)$ required for diHCF4-stabilized systems, but only at higher $w$ values (Table 2 ). Such critical-type behavior has been noted in hydrocarbon-based microemulsions on nearing the high temperature phase boundary. ${ }^{35,37}$ Clearly (Fig. 1) for higher $w$ value diHCF4stabilized F-MEs at $40{ }^{\circ} \mathrm{C}$, the high- $T$ boundary is much closer than that for the equivalent diCF3-stabilized samples. Hence, with reference to the phase behavior, the presence of attractive interactions is unsurprising.

The effect of surfactant concentration is probed for diCF3MEs at $w=40$ (Fig. 4, Fig. S6 $†$ ). From the fitted parameters (Table 1), it is clear that lowering surfactant concentration results in a higher polydispersity (and lower $R_{\mathrm{c}}-$ see eqn (2)). This effect can also be directly observed in the raw data (Fig. 4); the decrease in gradient around $Q=0.10 \AA^{-1}$ with decreasing [diCF3], accompanied with a less well-defined turnaround point at $Q \approx$ $0.02-0.03 \AA^{-1}$, is indicative of an increase in polydispersity. As these experiments were carried out at a constant OFP : solvent ratio, lowering [diCF3] effectively increases the ratio OFP :

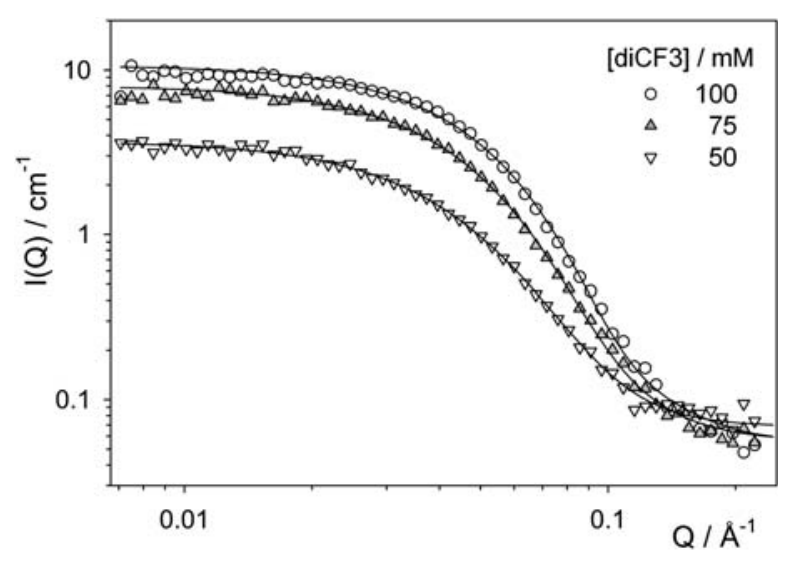

Fig. 4 Core-contrast SANS data from $\mathrm{D}_{2} \mathrm{O} / \mathrm{diCF} 3 / \mathrm{OFP} / \mathrm{HPFH}$ microemulsions at different [diCF3]. Solid lines represent form factor fits to the Schultz polydisperse spheres model. $w=40$, OFP $: H P F H=1: 9(\mathrm{v} / \mathrm{v})$, $T=40{ }^{\circ} \mathrm{C}$.

diCF3. Previously, for hydrocarbon-based AOT-stabilized systems with butanol co-surfactant, ${ }^{36,38}$ an increase in $p$ (and decrease in $R_{\mathrm{c}}$ ) was reported upon proportional increase in butanol content. The results here in F-based systems follow a similar trend seen with those hydrocarbon analogues.

The F-solvent type (HPFP or HPFH), already observed to have only minor effects on the phase behavior of the systems, also has little effect on SANS. The $R_{\mathrm{c}} v s . w$ swelling plot for diCF3/HPFH MEs is shown in the ESI $\dagger$ (Fig. S8). The calculated $a_{\mathrm{h}}\left(102 \pm 10 \AA^{2}\right)$ is close to those of other systems already discussed, indicating very similar interfacial absorption and packing across this series.

\section{Nanoparticle synthesis}

Using diCF3/HPFP F-MEs, silver nanoparticles (Ag-NPs) were synthesized, as detailed in the Experimental section. For comparison, the same synthesis was performed in a direct hydrocarbon analogue H-AOT-stabilized water-in-isooctane system. ${ }^{39}$ Upon addition of hydrazine to clear, colorless $\mathrm{Ag}^{+}$containing microemulsions, a color change to light yellow-green was observed, consistent with the formation of Ag-NPs (Fig. 5, top LHS). The large peak dominating the UV spectrum (Fig. 5, top RHS), at $\lambda_{\max } \approx 400 \mathrm{~nm}$ is consistent with the presence of Ag-NPs ${ }^{39-41}$ Some sedimentation was seen for diCF3-stabilized NPs, but not observed in the H-AOT system, which explains the difference in the absolute value of $A_{\max }$ between the two spectra. This reduced stability of diCF3-stabilized Ag-NP dispersions compared to the $\mathrm{H}$-carbon analogue is not surprising, given weaker intermolecular interactions in F-solvents when compared to hydrogenated solvents (e.g. isooctane).

Transmission electron microscopy (TEM) images (Fig. 5, bottom panels) show the Ag-NPs to be spherical with a wide size distribution from $30-120 \AA$. The polydispersity of the diCF3stabilized NPs is particularly high, as might be expected given the already observed high microemulsion polydispersity (SANS results). High-resolution TEM (HR-TEM), coupled with selected area electron diffraction (SAED), has been employed to investigate the crystalline nature of the Ag-NPs. Lattice separations (HR-TEM image; fringe separation $=1.5 \AA$, theoretical 


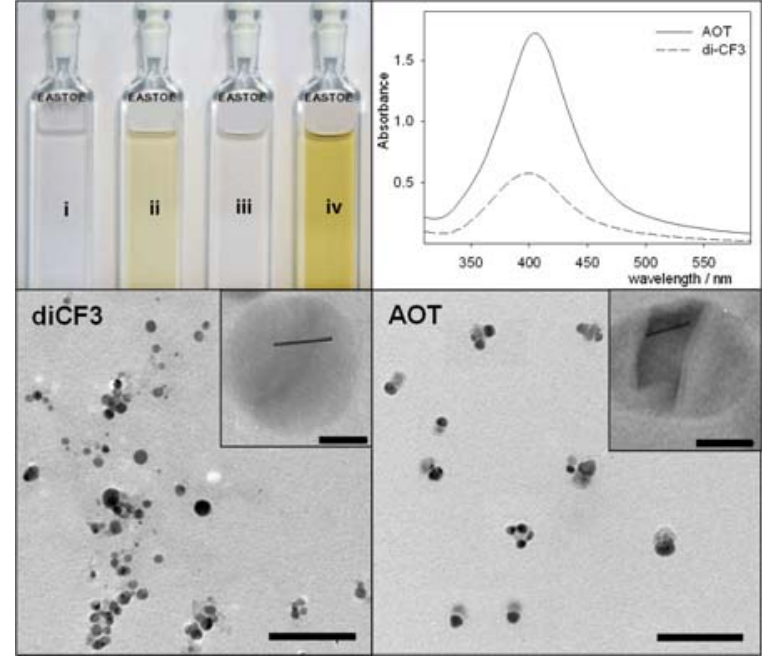

Fig. 5 (Top LHS) Visual appearance of (i) $\mathrm{AgNO}_{3}$-containing diCF3stabilized microemulsion in $2 \mathrm{H}, 3 \mathrm{H}$-perfluoropentane, (ii) diCF3-stabilized Ag-NPs in 2 $\mathrm{H}, 3 \mathrm{H}$-perfluoropentane, (iii) $\mathrm{AgNO}_{3}$-containing AOTstabilized microemulsion in isooctane, (iv) AOT-stabilized Ag-NPs in isooctane. All microemulsions were $w=10$, [surfactant] $=100 \mathrm{mM}$, $\left[\mathrm{Ag}^{+}\right]=1 \mathrm{mM}$. (Top RHS) UV spectra from solutions (ii) and (iv) in top left panel (diCF3- and AOT-stabilized nanoparticle dispersions). (Bottom panels) TEM and HR-TEM images (inset) of diCF3 (bottom LHS) and AOT (bottom RHS) coated Ag nanoparticles. Note: lattice separations (indicated by fringe separation) are highlighted on HR-TEM images. Scale bars are $500 \AA$ for TEM images, 50 A for HR-TEM images (inset).

value for $\mathrm{Ag}^{0}$ is $1.4 \AA$ ) and d-spacings (SAED: Fig. S9, Table $\mathrm{S} 2 \dagger$ ) obtained are consistent with an $\mathrm{Ag}^{0}$ cubic structure. ${ }^{42}$

\section{Nanoparticle recovery and redispersion}

An aliquot (5 ml) of a diCF3-stabilized Ag-NP dispersion was placed in a vacuum oven at $40^{\circ} \mathrm{C}$ for $48 \mathrm{~h}$. The resulting powder was light green, suggesting that the nanoparticles had not undergone extensive growth or fusion. This powder was dispersed in a $1: 9 \mathrm{v} / \mathrm{v}$ mixture of OFP : $\operatorname{HPFP}(5 \mathrm{ml})$ and filtered. The UV-vis spectrum (Fig. S10†) still shows an absorbance maximum at $\lambda=400 \mathrm{~nm}$, indicating some redispersed $\mathrm{Ag}$ nanoparticles. The process, however, is inefficient: comparing $A$ $\left(\lambda_{400 \mathrm{~nm}}\right)$ obtained for the original dispersion $(>0.5)$ with that of the recovered and then redispersed system $(<0.04)$ suggests that less than $10 \%$ of the original Ag-NPs were redispersed. However, it should be noted that diCF3 has low solubility in dry OFP/ HPFP and this may go some way towards explaining the discrepancy. Clearly a more sophisticated recovery method, such as colloidal separation or centrifugation might result in more efficient AgNP recovery. The ability to recover and redisperse NPs, and transfer them between different solvents may prove to be crucial for potential applications in catalysis.

\section{Experimental section}

\section{Chemicals}

$1 H, 1 H, 5 H$-Octafluoropentan-1-ol (OFP), $1 H$-perflourohexane (HPFH) and $2 \mathrm{H}, 3 \mathrm{H}$-perfluoropentane (HPFP) were purchased from Fluorochem Ltd and used without further purification. The surfactants sodium $\operatorname{bis}(1 H, 1 H, 5 H$-octafluoropentyl)-2-sulfosuccinate (diHCF4) and sodium bis $(1 H, 1 H$-perfluorobutyl)-2sulfosuccinate (diCF3) were synthesized and purified as detailed elsewhere. ${ }^{43,44}$ Hydrazine $\left(\mathrm{N}_{2} \mathrm{H}_{4}\right)$ and silver nitrate $\left(\mathrm{AgNO}_{3}\right)$ were purchased from Aldrich. Sodium bis(2-ethylhexyl)sulfosuccinate (AOT, Aldrich) was purified before use by Soxhlet extraction (dry ethyl acetate), followed by repeated centrifugation of solutions made up in dry methanol to remove any excess salt. ${ }^{33,34}$

\section{SANS}

SANS experiments were carried out on the time-of-flight LOQ instrument at ISIS, UK where incident wavelengths are $2.2 \leq \lambda \leq$ $10 \AA$, resulting in an effective $Q$ range of $0.009-0.249 \AA^{-1}$. Absolute intensities $( \pm 5 \%)$ for $I(Q)\left(\mathrm{cm}^{-1}\right)$ were determined by calibrating the received signal for a partially deuterated polymer standard. Measurements were carried out in single-phase microemulsion regions at $40{ }^{\circ} \mathrm{C}$, controlled by a thermostatted bath and carefully monitored throughout the experiments. Due to the similar scattering length densities (Table S1 $\dagger$ ) of the Fsolvents, F-surfactants and F-alcohol co-surfactant, only water core-contrast measurements could be performed. In the first instance, $\mathrm{D}_{2} \mathrm{O}$ was employed (inside microemulsion droplets), although some runs were repeated with $\mathrm{H}_{2} \mathrm{O}$ in place of $\mathrm{D}_{2} \mathrm{O}$ in an attempt to generate additional structural information. Some runs (Fig. S4 $\dagger$ ) were also repeated with freshly made samples to check reproducibility. Data were fitted to a polydisperse sphere form factor obeying a Schultz distribution, discussed in ESI $\dagger$, using the FISH iterative fitting program..$^{45}$ It should be noted that many different fitting models were assessed, including alternative $S(Q)$ functions; none of these gave adequate statistical fits, nor physically realistic descriptions. For some diHCF4 SANS data, it was found beneficial to include a small OrsteinZernike type attractive $S(Q)$ contribution. This is likely to be owing to the proximity of those particular samples to the high temperature microemulsion failure phase boundary.

\section{Nanoparticle synthesis}

Silver nanoparticles, stabilized by either AOT or diCF3, were formed by the reduction of microemulsified $\mathrm{AgNO}_{3}$ by hydrazine. The Ag-containing microemulsions were prepared at $w=$ 10, $[\text { surfactant }]_{\text {total }}=100 \mathrm{mM}$, and $\left[\mathrm{AgNO}_{3}\right]_{\text {total }}=1 \mathrm{mM}$. Hydrazine $\left(3 \mathrm{mM}\right.$, i.e. $\mathrm{Ag}^{+}$: hydrazine molar ratio $\left.=1: 3\right)$ was added to the microemulsions directly (dropwise, with vigorous stirring) using a Hamilton syringe. Samples were then stirred for $24 \mathrm{~h}$. UV spectra of the Ag-NPs were obtained after $24 \mathrm{~h}$ using a Thermo Evolution $300 \mathrm{UV}$-vis spectrometer. TEM images were obtained with a JEOL JEM 1200 EX Mk 2 having a standard Wfilament at $120 \mathrm{kV}$, fitted with a MegaViewIII digital camera, using Soft Imaging Systems GmbH analySIS 3.0 image analysis software. HR-TEM micrographs were obtained using a JEOL JEM 2010 microscope operating at $200 \mathrm{kV}$. Micrographs and SAED images were recorded on Kodak Electron Image Film SO163 producing very high-resolution negatives and processed using recommended Kodak solutions. 


\section{Conclusions}

A set of new four-component fluoro-sulfosuccinate-stabilized water-in-fluorocarbon microemulsions (F-MEs) has been developed and characterized. The influence of system composition on temperature-dependent phase behavior and nanodroplet structure has been investigated by neutron scattering, representing one of the first in-depth studies on F-microemulsion structure by SANS. Interestingly and significantly, these F-MEs stabilized by fluorinated analogues of AOT are found to behave in a very similar way to other AOT-based water-in-hydrocarbon oil, ${ }^{33-38}$ water-in- $\mathrm{CO}_{2}$ (e.g. ref. 25) and water-in-high-pressure fluorocarbon systems, ${ }^{15}$ highlighting the universality of the sulfosuccinate surfactant template as a stabilizing moiety for water microemulsion droplets in a wide variety of non-polar solvents. Silver nanoparticles have successfully been synthesized in diCF3stabilized F-MEs, and to the best of our knowledge this appears to represent the first synthesis of F-capped NPs using F-microemulsions as reaction media. The Ag-NPs have been thoroughly characterized by UV-vis spectrometry, transmission electron microscopy, single-particle energy dispersive X-ray analysis, selected area electron diffraction and high-resolution transmission electron microscopy. Ag-NPs have also been recovered and redispersed in the fluorinated solvent mixture. These results introduce a well-defined family of w/fc microemulsions suitable for applications and further study. They also provide a much needed link between high-pressure $\left(\mathrm{CO}_{2}\right.$ and $\left.\mathrm{fc}\right)$ and ambient liquid hydrocarbon microemulsion systems. The ability to generate and stabilize NPs in these fluorous systems suggests applications in fluorocarbon-based catalysis, or possibly in drug delivery. In addition, given the known high $\mathrm{CO}_{2}$ compatibility of F-compounds, ${ }^{30-32}$ this w/fc microemulsion-based method provides an alternative pathway to obtain F-capped NPs, compared to existing approaches. ${ }^{24}$

\section{Acknowledgements}

Jon Jones and John Mitchells are thanked for their help with the generation of HR-TEM micrographs. MJH and KJM thank EPSRC, Kodak, and School of Chemistry at the University of Bristol for the provision of PhD scholarships. The ISIS-STFC Neutron Scattering Facility (formerly CCLRC) is thanked for the provision of beam-time and grants towards consumables and travel. The EPSRC is thanked for provision of funding under grants EP/C523105/1 and EP/F020686.

\section{Notes and references}

1 R. W. Marla, S. G. Jay and H. S. Thomas, Pediatr. Pulmonol., 1998, 26, 42-63.

2 M. J. Lawrence and G. D. Rees, Adv. Drug Delivery Rev., 2000, 45, 89-121.

3 V. M. Sadtler, M. P. Krafft and J. G. Riess, Colloids Surf., A: Physicochemical and Engineering Aspects, 1999, 147, 309-315.

4 M. P. Krafft, A. Chittofrati and J. G. Riess, Curr. Opin. Colloid Interface Sci., 2003, 8, 251-258.

5 J. G. Riess, Tetrahedron, 2002, 58, 4113-4131.

6 H. M. Courrier, T. F. Vandamme and M. P. Krafft, Colloids Surf., A: Physicochemical and Engineering Aspects, 2004, 244, 141-148.

7 K. Debbabi, F. Guittard, J. Eastoe, S. Rogers and S. Geribaldi, Langmuir, 2009, 25, 8919-8926.
8 N. Patel, M. Marlow and M. J. Lawrence, J. Colloid Interface Sci., 2003, 258, 345-353.

9 A. Robert and C. Tondre, J. Colloid Interface Sci., 1984, 98, 515-522.

10 P. LoNostro, S.-M. Choi, C.-Y. Ku and S.-H. Chen, J. Phys. Chem. $B, 1999,103,5347-5352$.

11 N. Patel, M. Marlow and M. J. Lawrence, J. Colloid Interface Sci., 2003, 258, 354-362.

12 C. M. C. Gambi, R. Giordano, M. Laurati, L. Lanzi, F. Pini and P. Baglioni, Appl. Phys. A, 2002, 74, s377-s379.

13 P. Baglioni, C. M. C. Gambi, R. Giordano and D. Senatra, J. Mol. Struct., 1996, 383, 165-169.

14 P. Baglioni, C. M. C. Gambi and R. Giordano, Physica B (Amsterdam), 1997, 234-236, 295-296.

15 D. C. Steytler, M. Thorpe, J. Eastoe, A. Dupont and R. K. Heenan, Langmuir, 2003, 19, 8715-8720.

16 I. T. Horvath and J. Rabai, Science, 1994, 266, 72-75.

17 T. Mathivet, E. Monflier, Y. Castanet, A. Mortreux and J.-L. Couturier, Tetrahedron, 2002, 58, 3877-3888.

18 H. R. Hobbs and N. R. Thomas, Chem. Rev., 2007, 107, 2786-2820.

19 M. Boutonnet, S. Lögdberg and E. Elm Svensson, Curr. Opin. Colloid Interface Sci., 2008, 13, 270-286.

20 M. D. Hughes, Y.-J. Xu, P. Jenkins, P. McMorn, P. Landon, D. I. Enache, A. F. Carley, G. A. Attard, G. J. Hutchings, F. King, E. H. Stitt, P. Johnston, K. Griffin and C. J. Kiely, Nature, 2005, 437, 1132-1135.

21 J. Eastoe, M. J. Hollamby and L. Hudson, Adv. Colloid Interface Sci., 2006, 128-130, 5-15.

22 M.-P. Pileni, Nat. Mater., 2003, 2, 145-150.

23 B. L. Cushing, V. L. Kolesnichenko and C. J. O'Connor, Chem. Rev., 2004, 104, 3893-3946.

24 P. S. Shah, J. D. Holmes, R. C. Doty, K. P. Johnston and B. A. Korgel, J. Am. Chem. Soc., 2000, 122, 4245-4246.

25 J. Eastoe, S. Gold and D. C. Steytler, Langmuir, 2006, 22, 9832-9842.

26 X. Dong, D. Potter and C. Erkey, Ind. Eng. Chem. Res., 2002, 41, 4489-4493.

27 P. G. Rogueda, Drug Dev. Ind. Pharm., 2003, 29, 39-49.

28 P. Selvam, R. P. S. Peguin, U. Chokshi and S. R. P. da Rocha, Langmuir, 2006, 22, 8675-8683.

29 J. Eastoe, A. Downer, A. Paul, D. C. Steytler, E. Rumsey, J. Penfold and R. K. Heenan, Phys. Chem. Chem. Phys., 2000, 2, 5235-5242.

30 J. B. McClain, D. E. Betts, D. A. Canelas, E. T. Samulski, J. M. DeSimone, J. D. Londono, H. D. Cochran, G. D. Wignall, D. Chillura-Martino and R. Triolo, Science, 1996, 274, 2049-2052.

31 A. I. Cooper, J. D. Londono, G. Wignall, J. B. McClain, E. T. Samulski, J. S. Lin, A. Dobrynin, M. Rubinstein, A. L. C. Burke, J. M. J. Frechet and J. M. DeSimone, Nature, 1997, 389, 368-371.

32 M. Z. Yates, D. L. Apodaca, M. L. Campbell, T. M. McCleskey and E. R. Birnbaum, Chem. Commun., 2001, 25-26.

33 S. Nave, PhD thesis, University of Bristol, Bristol, 2001.

34 S. Nave, J. Eastoe, R. K. Heenan, D. C. Steytler and I. Grillo, Langmuir, 2000, 16, 8741-8748.

35 M. Kotlarchyk, S.-H. Chen and J. S. Huang, Phys. Rev. A, 1983, 28, 508-511.

36 E. Zambrano, M. Kotlarchyk, A. Langner and A. Faraone, J. Phys.: Condens. Matter, 2006, 18, S2451-S2466.

37 M. Kotlarchyk, S.-H. Chen, J. S. Huang and M. W. Kim, Phys. Rev. A, 1984, 29, 2054-2069.

38 B. Farago, D. Richter, J. S. Huang, S. A. Safran and S. T. Milner, Phys. Rev. Lett., 1990, 65, 3348-3351.

39 P. W. Bell, M. Anand, X. Fan, R. M. Enick and C. B. Roberts, Langmuir, 2005, 21, 11608-11613.

40 M. Anand, P. W. Bell, X. Fan, R. M. Enick and C. B. Roberts, J. Phys. Chem. B, 2006, 110, 14693-14701.

41 C. Petit, P. Lixon and M.-P. Pileni, J. Phys. Chem., 1993, 97, 12974 12983.

$42 W W W$-MINCRYST, SILVER-4219. WWW-MINCRYST, 2008. Crystallographic and Crystallochemical Database for minerals and their structural analogues http://database.iem.ac.ru/mincryst.

43 N. Yoshino, N. Noboru Komine, J. Suzuki, Y. Arima and H. Hirai, Bull. Chem. Soc. Jpn., 1991, 64, 3262-3266.

44 A. Downer, J. Eastoe, A. R. Pitt, E. A. Simister and J. Penfold, Langmuir, 1999, 15, 7591-7599.

45 R. K. Heenan, RAL-89-129, Didcot, UK, 1989. 